\title{
Détermination de la qualité des eaux du littoral mauritanien par la mesure des métaux traces chez les mollusques Donax rugosus et Venus verrucosa
}

\section{Assessment of water quality on the mauritanian coast by measurement of trace metals in Donax rugosus and Venus verrucosa molluscs}

Sidoumou $Z^{\star}$, Roméo M.**, Gnassia-Barelli M.**, Nguyen Ph.* et Caruba R. ${ }^{\star \star \star}$

* ISS, BP 5026, Nouakchott, Mauritanie.

** INSERM, Unité 303 "Mer et Santé", Laboratoire de Toxicologie marine, Faculté de Médecine, 06107 Nice Cedex 2.

*** Université de Nice Sophia Antipolis, Laboratoire de Géologie et Géochimie, Parc Valrose, 06034 Nice Cedex, France.

Résumé. - Les variations des concentrations en $\mathrm{Cd}, \mathrm{Cu}, \mathrm{Fe}, \mathrm{Mn}$ et $\mathrm{Zn}$ ont été analysées chez deux Mollusques prélevés l'un le long de la côte mauritanienne (Donax rugosus) et l'autre dans deux baies du nord de la Mauritanie (Venus verrucosa). Pour $D$. rugosus, les poids variant, nous avons examiné les relations $C=a P^{b}, C=$ concentration en métal, $\mathrm{P}=$ poids $\mathrm{sec}$ et $\mathrm{b}=$ coefficient de régression. Dans tous les cas, le coefficient b est négatif. Pour $V$. verrucosa, les poids varient peu, $V$. verrucosa, animal le plus gros, a les concentrations les plus faibles en métaux, à l'exception du cadmium.

Mots-clés. - Littoral mauritanien, Métaux traces, Mollusques, Venus verucosa, Donax rugosus.

Ce travail a été réalisé dans le cadre d'un accord inter-universitaire: Université de Nice, Institut Supérieur des Sciences de Nouakchott. 
Abstract. - Variations of metal concentrations ( $\mathrm{Cd}, \mathrm{Cu}, \mathrm{Fe}, \mathrm{Mn}$ and $\mathrm{Zn}$ ) were determined in two molluscs collected from the Mauritanian coast (Donax rugosus) and from two Northern bays of Mauritania (Venus verrucosa). As the weight of $D$. rugosus was varying, the relationships: $C=a W^{b}$ were examined where $C$ is the metal concentration in the organism, $W$ the dry weight and $b$ the regression coefficient. In all cases, the coefficient b was negative. As regards $V$. verrucosa, weights did not vary much. Comparison of trace-metal concentrations in the small $D$. rugosus and the large $V$. verrucosa revealed lower contents in $V$. verrucosa, except for cadmium.

Key words. - Mauritanian coast, Trace metals, Molluscs, Venus verrucosa, Donax rugosus.

La production biologique du plateau continental mauritanien est l'une des plus riches du monde. C'est une zone d'«upwelling" intense, permanent en ce qui concerne le cap Blanc et saisonnier plus au sud au niveau de Nouakchott. Ceci lui permet de pratiquer un effort de pêche sans cesse croissant. Une surveillance constante de la qualité des produits marins (en particulier la concentration en métaux traces potentiellement polluants) destinés à la consommation locale ainsi qu'à l'exportation est donc nécessaire (Roméo, 1987, Roméo et Gnassia-Barelli, 1988 et Sidoumou, 1991).

Les espèces «indicatrices" choisies pour assurer cette surveillance sont deux mollusques benthiques, particulièrement abondants sur les côtes mauritaniennes: le "haricot de mer » Donax rugosus et la Praire Venus verrucosa. $D$. rugosus vit dans des sédiments de l'étage médiolittoral alors que $V$. verrucosa dans ceux de l'étage infra-littoral.

\section{MATÉRIEL ET MÉTHODES}

Les figures 1 et 2 montrent les lieux de prélèvement de Venus verrucosa et de Donax rugosus, la figure 2 détaillant les zones de collecte de $D$. rugosus du nord de Nouakchott au sud de la Mauritanie. Les échantillons de Donax rugosus ont été récoltés à la main, à marée basse en douze stations le long de $260 \mathrm{~km}$ de côte entre N'Diago (7 stations situées à distance croissante de 100 en $100 \mathrm{~m}$ de l'embouchure du fleuve Sénégal) et Tanit (40 km au nord de Nouakchott) lors d'une campagne réalisée en juin 1989. Après la récolte, les Donax sont placés pendant $24 \mathrm{~h}$ dans de l'eau de mer propre afin de vider leur tube digestif. Les prélèvements de Venus verrucosa ont été réalisés à partir du N/O Almoravide (appartenant au CNROP) à l'aide d'une drague à praires, au nord de la Mauritanie en deux stations: la station "baie du Lévrier" et la station "baie de Cansado" (celle-ci se trouve à l'intérieur de

Fig. 1. - Localisation des aires de prélèvements de Donax et de Praires sur la côte mauritanienne.

Fig. 1. - Location of the Donax and clam sampling area on the Mauritanian coast. 
Mesure des métaux traces chez les mollusques

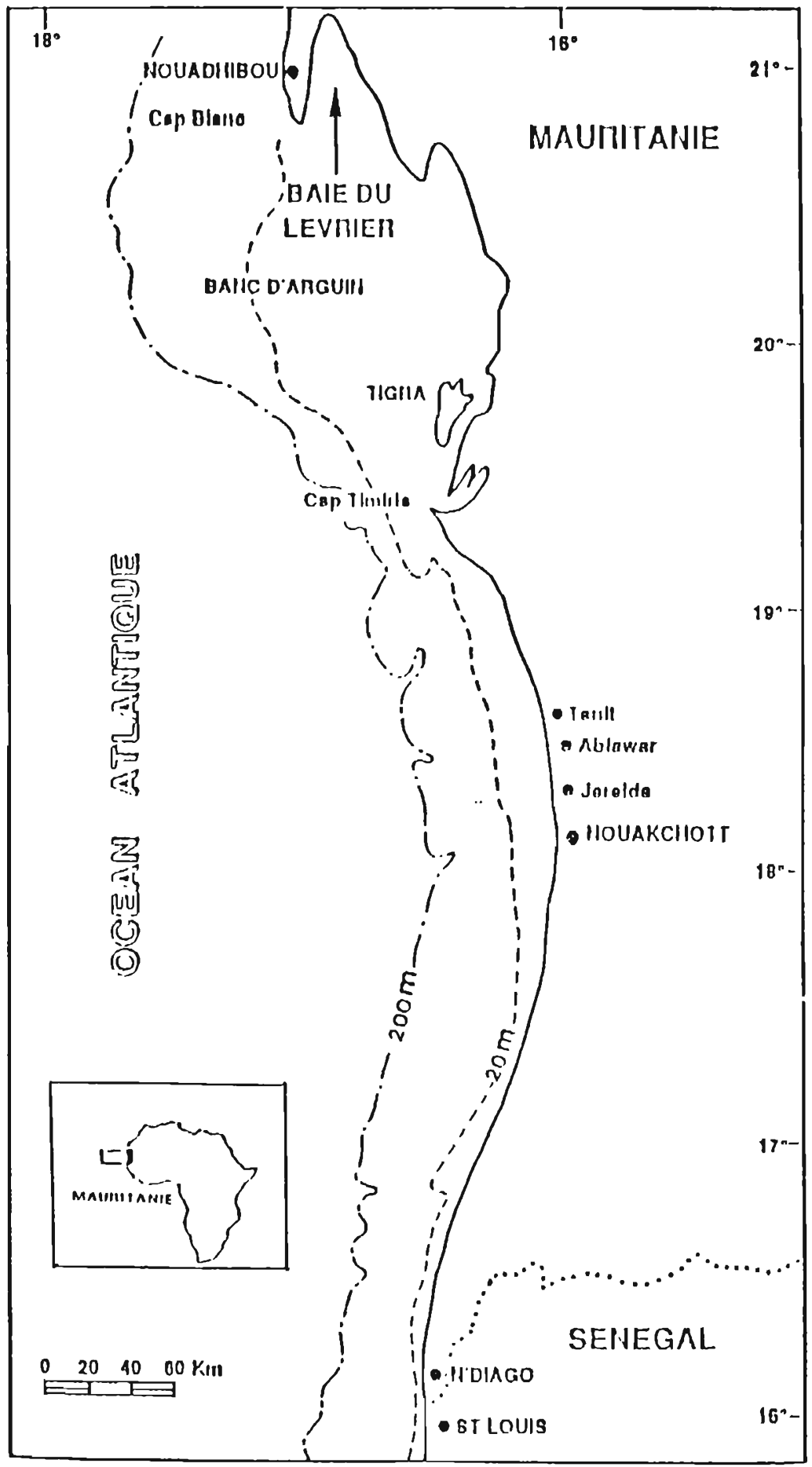


la baie du Lévrier, fig. 1), entre février 1987 et septembre 1988.

En vue de l'analyse en métaux, les parties molles des animaux sont minéralisées avec de l'acide nitrique suprapur à $65 \%$ dans un four microondes programmable. Le minéralisat est analysé en spectrophotométrie d'absorption atomique en flamme pour $\mathrm{Cu}, \mathrm{Fe}, \mathrm{Mn}$ et $\mathrm{Zn}$ et en four pour Cd.

\section{RÉSULTATS}

Les Donax ayant des poids nettement différents, nous nous sommes servis, pour examiner les variations des concentrations en métaux de la relation: concentration en métal en fonction du poids sec des tissus mous, donnée par Boyden (1974, 1977). Cette relation est de la forme: $\mathrm{C}=\mathrm{a}$ $\mathrm{P}^{\mathrm{b}}$ où $\mathrm{C}=$ concentration en métal dans l'organisme $\left(\mu \mathrm{g} \cdot \mathrm{g}^{-1}\right), P=$ poids $\sec (g), a=$ ordonnée à l'origine, $b=$ coefficient de régression. Nous avons déterminé les relations concentra- tion/poids pour chaque métal. Ces relations sont données dans le tableau 1, tous sites confondus. Puis, nous avons groupé, pour des animaux de même poids, les stations en fonction de leur situation géographique: les 7 stations du sud (N'Diago), les 2 stations du centre près de Nouakchott et les 3 stations au nord de Nouakchott (Tanit). En passant du sud au nord, les concentrations moyennes en $\mathrm{Cd}$ diminuent de 1,2 à $0,5 \mu \mathrm{g} \cdot \mathrm{g}^{-1}$. Nous remarquons, en revanche, une tendance à l'augmentation des concentrations moyennes en $\mathrm{Cu}$ et Mn du sud $\left(14,9 \mu \mathrm{g} \mathrm{Cu} \mathrm{g}^{-1}\right.$ et $7,9 \mu \mathrm{g}$ $\left.\mathrm{Mn} \mathrm{g}^{-1}\right)$ au nord $\left(27,8 \mu \mathrm{g} \mathrm{Cu} \mathrm{g}^{-1}\right.$ et $21,1 \mu \mathrm{g} \mathrm{Mn} \mathrm{g}^{-1}$ ). Les concentrations en fer sont nettement plus faibles (de l'ordre de $250 \mu \mathrm{g} \mathrm{g}^{-1}$ ) pour les animaux provenant des stations du centre par rapport à celles des animaux prélevés au sud (686 $\mathrm{g} \mathrm{g} \mathrm{g}^{-1}$ ) ou au nord $\left(784 \mu \mathrm{g} \mathrm{g}^{-1}\right)$. Les variations des concentrations en zinc chez $D$. rugosus sont faibles en passant du sud $\left(103 \mu \mathrm{g} \mathrm{g}^{-1}\right)$ au nord $\left(127 \mu \mathrm{g} \mathrm{g}^{-1}\right)$.

Pour les concentrations en traces métalliques des Praires, étant donné

Tableau 1. - Relations entre les concentrations corporelles en métaux et le poids sec chez Donax rugosus C (concentration en métal en $\left.\mu \mathrm{g}^{-1} \mathrm{~g}^{-1}\right)=\mathrm{a} \mathrm{P}^{\mathrm{b}}, \mathrm{P}=$ poids $\sec (\mathrm{en} \mathrm{g}$ ), $r=$ coefficient de corrélation.

Table 1. - Relationships between the metal content and the body dry weight in Donax rugosus.

\begin{tabular}{l|c|c|}
\multicolumn{1}{c|}{ Equations } & Erreurs sur b & \\
\hline Ensemble des stations de prélèvement $(n=116$ échantillons) & \\
$\mathrm{Cd}=0,76 \mathrm{P}^{-0,26}$ & 0,10 & 0,577 \\
$\mathrm{Cu}=14 \mathrm{P}^{-0.43}$ & 0,06 & 0,644 \\
$\mathrm{Fe}=415 \mathrm{P}^{-0,56}$ & 0,07 & 0,520 \\
$\mathrm{Mn}=11 \mathrm{P}^{-0,08}$ & 0,08 & 0,716 \\
$\mathrm{Zn}=106 \mathrm{P}^{-0,15}$ & 0,05 & -1 \\
\hline
\end{tabular}


que les animaux prélevés avaient un poids sec peu variable, nous avons pu comparer les concentrations en métaux (et non plus les relations $C=a P^{b}$ ) selon le lieu de prélèvement (tableau 2). Les concentrations en cuivre, cadmium et zinc sont plus faibles (test $t$ de comparaison entre les moyennes significatif)dans la baie de Cansado que dans la baie du Lévrier. Pour le fer et le manganèse, les moyennes ne présentent pas de différences significatives entre les deux baies.

Pour savoir si les animaux absorbent les éléments traces étudiés ou si ces derniers sont simplement adsorbés (souvent sur les branchies) ou contenus dans le tube digestif, nous avons effectué des expériences d'épuration sur des lots de Donax rugosus de même taille prélevés le 9 janvier 1990 au Cabanon du colonel. Ces lots ont été divisés en deux groupes: le premier groupe $(n=10)$ a été congelé quelques heures après le prélèvement alors que le deuxième groupe $(n=10)$ a été congelé après un séjour d'une semaine dans de l'eau de mer. Les concentrations en métaux (exprimées en $\mu \mathrm{g} \cdot \mathrm{g}^{-1}$ ) du premier groupe et du second groupe sont respectivement pour le cadmium $0,89 \pm 0,10$ et $0,86 \pm 0,26$; pour le cuivre $13,56 \pm 0,34$ et $13,25 \pm 2,0$; pour le zinc $150 \pm 42$ et $124 \pm 3$; pour le fer $590 \pm 141$ et $113 \pm 12$ (soit une diminution de $80 \%$ ) et pour le manganèse $12,48 \pm 1,35$ et $5,19 \pm 0,70$ (soit une diminution de $58 \%)$.

\section{DISCUSSION}

Les relations concentrations en métaux traces/poids sec établies pour l'ensemble des échantillons de Donax prélevés à la même date le long de la côte mauritanienne donnent un b négatif. Selon Boyden (1974) si b est négatif, le métal est adsorbé par les tissus vivants des Mollusques, les animaux les plus petits auront les concentrations les plus fortes ou bien une absorption plus faible pour les organismes de plus grande taille en raison de leur niveau d'énergie métabolique inférieur (Blueweiss et

Tableau 2. - Concentrations en $\mathrm{Cd}, \mathrm{Cu}, \mathrm{Fe}, \mathrm{Mn}$ et $\mathrm{Zn}\left(\mu \mathrm{g} \cdot \mathrm{g}^{-1}\right.$ de poids sec) des Praires, Venus verrucosa, en deux stations du littoral mauritanien.

Table 2. - Metal content ( $\mu \mathrm{g} \cdot \mathrm{g}^{-1} \mathrm{dry}$ weight) in clams (Venus verrucosa) from two coves on the Mauritanian coast.

\begin{tabular}{|l|l|l|l|}
\hline Métaux & Cansado $(\mathbf{n}=39)$ & Lévrier $(\mathbf{n}=20)$ & Test t \\
\hline $\mathrm{Cd}$ & $3,93 \pm 2,84$ & $11,86 \pm 4,82$ & \\
$\mathrm{Cu}$ & $5,46 \pm 2,54$ & $7,07 \pm 3,03$ & $*$ \\
$\mathrm{Fe}$ & $277 \pm 154$ & $322 \pm 170$ & $\mathrm{NS}$ \\
$\mathrm{Mn}$ & $5,58 \pm 3,17$ & $3,92 \pm 1,47$ & $\mathrm{NS}$ \\
$\mathrm{Zn}$ & $48 \pm 20$ & $64 \pm 18$ & $\vdots$ \\
\hline
\end{tabular}

NS non significatif, " $\mathrm{t}$ significatif à $\alpha=0,02,{ }^{*} \alpha=0,01, \cdots * \alpha=0,001$. 


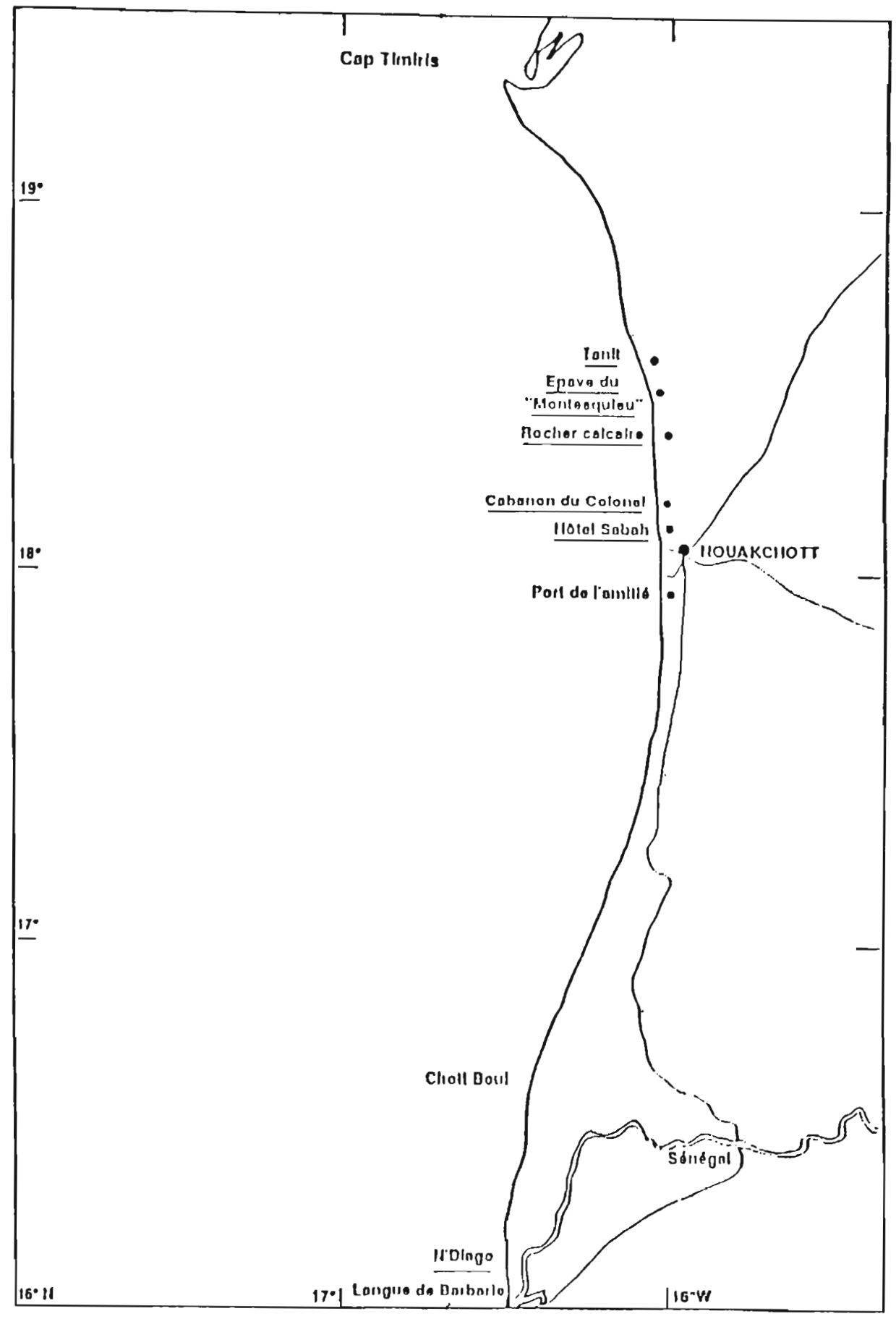

Fig. 2. - Localisation détaillée des aires de prélevement des Donax le long de la côte mauritanienne.

Fig. 2 - Detailed location of sampling areas along the Mauritanian coast. 
al., 1978). Des concentrations plus élevées en cadmium (b positif) ont été rapportées par Boyden (1977) chez des Mollusques adultes Patella vulgata par rapport aux animaux plus jeunes. De plus, un coefficient $b$ de 1,16 est noté pour le cadmium chez des moules matures par Berthet (1986) qui émet l'hypothèse que lorsque la croissance ralentit, si le flux de métal reste constant, sa concentration augmente chez l'animal.

Au sud l'industrialisation de la région de l'embouchure du fleuve Sénégal expliquerait les fortes concentrations en cadmium. Cossa et al. (1980) ont trouvé une modification de la relation taille (et donc poids)concentration en $\mathrm{Cd}$ selon les sites de prélèvement chez Mytilus edulis. L'augmentation des concentrations en cuivre et en manganèse chez les animaux est peut être due à celle de la concentration en métaux dans les eaux. Peut-être l'intensité des "upwelling" vers le nord influence-t-elle ce phénomène? Les eaux des "upwelling " sont généralement riches en ces métaux traces (Bruland et Franks, 1983). Les expériences de dépuration effectuées sur Donax rugosus ont montré que le fer présent dans ces animaux était soit faiblement adsorbé à l'animal soit présent dans son tube digestif. On peut penser que, du fait des parametres hydrologiques caractérisant les stations du centre, le fer total (soluble et particulaire) présent dans l'eau de mer, à ces stations, serait moins disponible pour l'accumulation. En effet, les eaux de cette région sont très renouvelées à cause du dé- ferlement permanent des vagues (barre) et la forme chimique ou la concentration du fer total peuvent changer. Pour le zinc, les concentrations varient peu, ce métal semblerait donc être bien régulé.

Les Praires ont été prélevées dans une zone de grande fertilité due à un upwelling permanent, situé au niveau du Cap Blanc (fig. 1). Les concentrations en cadmium, cuivre, et zinc des Praires du centre de la baie du Lévrier sont plus élevées que celles des Praires de la baie du Cansado. Or, la baie de Cansado reçoit directement les déchets des deux ports (pêche, commerce), des industries de pêche, des égoûts de la ville de Nouadhibou et de la bourgade de Cansado. La baie de Cansado est aussi à proximité d'une raffinerie et d'un port minéralier. Les résultats peuvent alors s'expliquer par la courantologie de la région, en fait il y a un courant qui longe la côte et passe par l'entrée de la baie de Cansado alors que le centre de la baie du Lévrier, bien qu'assez éloigné des sources de rejets anthropiques, est une région où l'eau est calme, son renouvellement est beaucoup plus lent, et l'évaporation est intense. Les concentrations en fer et en manganèse des Venus verrucosa provenant des deux baies sont variables mais il n'y a pas de différence significative entre celles-ci, ce qui implique que les concentrations en fer total et en manganèse total dans l'eau ne sont pas différentes dans l'une et l'autre baies.

Les moyennes générales des concentrations en métaux, calculées sur un échantillon de 116 Donax et 
59 Praires, sont de $1,1 \mu \mathrm{g} \cdot \mathrm{g}^{-1} \mathrm{Cd}$, $20,6 \mu \mathrm{g} \cdot \mathrm{g}^{-1} \mathrm{Cu}, 658 \mu \mathrm{g} \cdot \mathrm{g}^{-1} \mathrm{Fe}$, $13,4 \mu \mathrm{g} \cdot \mathrm{g}^{-1} \mathrm{Mn}$ et $123 \mu \mathrm{g} \cdot \mathrm{g}^{-1} \mathrm{Zn}$ pour Donax rugosus et pour Venus verrucosa de $6,6 \mu \mathrm{g} \cdot \mathrm{g}^{-1} \mathrm{Cd}$, $6,0 \mu \mathrm{g} \cdot \mathrm{g}^{-1} \mathrm{Cu}, 292 \mu \mathrm{g} \cdot \mathrm{g}^{-1} \mathrm{Fe}$, $5,0 \mu \mathrm{g} \cdot \mathrm{g}^{-1} \mathrm{Mn}$ et $53 \mu \mathrm{g} \cdot \mathrm{g}^{-1} \mathrm{Zn}$. Bien que ces moyennes ne soient que des indications, nous constatons que l'espèce la plus grosse étudiée, Venus verrucosa, a les concentrations les plus faibles en métaux à l'exception du cadmium.

Si l'on compare les résultats des concentrations en métaux traces trouvés ici sur Donax rugosus et Venus verrucosa avec ceux donnés par différents auteurs pour les mollusques, on constate que la Praire Venus verrucosa a une grande capacité à concentrer le cadmium, métal nonessentiel. Par exemple, Amiard et al. (1986) donnent, pour les concentrations en cadmium de populations naturelles de moule Mytilus edulis prélevées en Atlantique, un domaine de variation de 0,52 à $1,79 \mu \mathrm{g} \mathrm{Cd} \mathrm{g}{ }^{-1}$ (moules de poids moyen de 0,223 g) et de 0,69 à 1,97 $\mu \mathrm{g} \mathrm{Cd} \mathrm{g}^{-1}$ (moules de poids moyen de $0,430 \mathrm{~g}$ ). Cossa (1988) trouve une moyenne de $0,8 \mu \mathrm{g}$ $\mathrm{Cd} \mathrm{g}^{-1}$ pour le genre Mytilus et un domaine de variation de 0,5 à 2,7 $\mu \mathrm{g} \mathrm{Cd}$ $\mathrm{g}^{-1}$ pour les moules Mytilus edulis provenant de différentes régions incluant plus de 600 stations à travers le monde. Cet auteur a, de plus, établi un modèle reliant in situ les teneurs en cadmium dans l'eau de mer et dans les moules. Mart et Nürnberg (1986) rapportent qu'en Atlantique les valeurs des concentrations en cad- mium, mesurées dans les eaux de surface sur une radiale entre Recife et Lisbonne, sont de l'ordre de $2 \mathrm{ng}$ $\mathrm{I}^{-1}$ sauf dans l'upwelling du Sénégal où les valeurs atteignent $16 \mathrm{ng} \mathrm{I}^{-1}$. Nous pouvons estimer que les eaux mauritaniennes auraient des concentrations élevées en cadmium du fait des remontées d'eaux profondes plus intenses en Mauritanie qu'au Sénégal, ce qui expliquerait les teneurs en cadmium élevées rencontrées chez la Praire, prélevée en zone d'upwelling permanent.

\section{RÉFÉRENCES BIBLIOGRAPHIQUES}

Amiard J.C., Amiard-Triquet C., Berthet B. et Métayer C., 1986. Contribution to the ecotoxicological study of cadmium, lead, copper and zinc in the mussel Mytilus edulis. I. Field study. Mar. Biol., 90: 425-431.

Berthet B., 1986. Études in situ et expérimentales du devenir de quelques éléments métalliques ( $\mathrm{Cd}, \mathrm{Pb}, \mathrm{Cu}$ et $\mathrm{Zn}$ ) dans un écosystème marin de zone conchylicole. Doc. d'État ès-Sciences, Scien. Biol., Université de Rennes I, $220 \mathrm{pp}$.

Blueweiss L., Fox H., Kudzma V., Nakashima D., Peters R. \& Sams S., 1978. Relationship between body size and some life history parameters. Oecologia (Berl.), 37 : 257-272.

Boyden C.R., 1974. Trace metal content and size in Molluscs. Nature (Long.), 251: 311-314.

Boyden C.R., 1977. Effect of size upon metal content of shellfish. J. mar. biol. Ass. UK, 57: 675-714. 
Bruland K.W. et Franks R.P., 1983. Mn, $\mathrm{Ni}, \mathrm{Cu}, \mathrm{Zn}$ and $\mathrm{Cd}$ in the Western North Atlantic. In: Trace metals in sea water. C.S. Wong, E. Boyle, K.W. Bruland, J.D. Burton and E.D. Goldberg (eds) : 395-414.

Cossa D., 1988. Cadmium in Mytilus spp.: worldwide survey and relationship between seawater and mussel content. Mar. Environ. Res. 26: 265-284.

Cossa D., Bourget E., Pouliot D., Piuzé J. et Chanut J.P., 1980. Geographical and seasonal variations in the relationship between trace metal content and body weight in Mytilus edulis. Mar. Biol., 58: 7-14.
Mart L. et Nürnberg H.W., 1986. The distribution of cadmium in the sea. Experientia, 50 suppl. : 28-40.

Roméo M., 1987. Trace metal in fish roe from the Mauritania coast. Mar. Pollut. Bull., 18: 507-508.

Roméo M. et Gnassia-Barelli M., 1988. Donax trunculus and Venus verrucosa as bioindicators of trace metal concentrations in Mauritanian coastal waters. Mar. Biol., 99: 223-227.

Sidoumou Z., 1991. Qualité des eaux du littoral mauritanien: étude des métaux traces chez deux mollusques bivalves Venus verrucosa et Donax rugosus. Doc. Sciences de la Vie. Université de Nice-Sophia Antipolis, 184 pp. 